\title{
Mental health stigma and help-seeking intentions in police employees
}

\author{
Daniel W. Grupe \\ Center for Healthy Minds, University of Wisconsin-Madison \\ 625 W Washington Ave, Madison, WI 53703 \\ 608-263-7572 grupe@wisc.edu
}

\begin{abstract}
$\underline{\text { Acknowledgements }}$
Thank you to our study participants and liaisons from each of our partner agencies, including the Madison Police Department (Captain Mindy Winter), the University of Wisconsin-Madison Police Department (Captain Mark Silbernagel), and the Fitchburg Police Department (Chief Chad Brecklin)
\end{abstract}

\section{Funding}

DWG was supported by a Career Development Award from the National Institute of Mental Health (K01MH117222)

$\underline{\text { Data availability statement }}$

Data will be provided by the author upon reasonable request 


\section{Mental health stigma and help-seeking intentions in police employees}

Mental health problems among police employees are exacerbated by negative attitudes and beliefs around mental health help-seeking perpetuated by police culture. We collected anonymous survey data from 259 civilian and commissioned police employees to test hypothesized relationships among help-seeking stigma, help-seeking attitudes, and intended help-seeking behavior. Results demonstrated that mental help-seeking stigma was negatively associated with help-seeking attitudes, and in turn with reduced mental health help-seeking intentions. Structural equation modeling provided support for a model linking help-seeking stigma, help-seeking attitudes, and intentions to seek help. This path model was moderated by psychological distress and previous participation in mindfulness training, which had opposing effects on help-seeking stigma and (indirectly) on intended help-seeking. These results support the hypothesis that stigma around mental health help-seeking contributes to reduced help-seeking intentions, and provide insight into policies, practices, and interventions that police agencies may enact to combat stigma and positively influence mental health help-seeking. 


\section{Mental health stigma and help-seeking intentions in police employees Introduction}

Recent data indicate that rates of mental illness among law enforcement professionals likely exceed those of the general population. A meta-analysis including data from over 270,000 police personnel found positive screening rates of $15 \%$ for posttraumatic stress disorder (PTSD), $14 \%$ for depression, $11 \%$ for generalized anxiety disorder, and $25 \%$ for hazardous drinking (Syed et al., 2020). A separate national survey of Canadian first responders found that $37 \%$ of municipal and provincial police officers, and 50\% of federal officers, screened positive for at least one mental health disorder (Carleton et al., 2017). Psychological distress is often accompanied by restricted attentional and self-regulatory capacity, elevated fatigue, and increased anger and aggression that can have a deleterious impact on police officers' ability to bring clear thinking, careful attention, and empathic, non-discriminatory responses to community members in their time of greatest need (Goff \& Rau, 2020; Ma et al., 2013; Rajaratnam et al., 2011). In short, promoting better mental health care among police employees is essential for the well-being of those inside the profession as well as that of the broader community.

These high rates of mental illness reflect demanding job responsibilities that include routine exposure to trauma and other forms of human suffering (Weiss et al., 2010), but there is also a clear part that police culture plays in this mental health crisis (Tuckey et al., 2012). Karaffa \& Tochkov (2013) identified three specific values that are highly esteemed in police culture: masculinity, independence, and emotional control. Police officers are socialized to not openly discuss the emotions they experience at work (particularly emotions related to personal suffering), and are made to believe that an inability to maintain control over one's emotions can get in the way of decision-making and peak performance, or jeopardize one's career prospects 
(Karaffa \& Koch, 2016; Karaffa \& Tochkov, 2013; Tuckey et al., 2012). Acknowledging emotional difficulties, or offering emotional support to those in need, runs contrary to traditional ideals of masculinity that persist in the profession and can lead to shame or ridicule by one's peers (Pasciak \& Kelley, 2013). The value of independence dissuades police officers from seeking help for mental health challenges, which may be seen as a sign of weakness and lead peers and supervisors to question an officer's fitness for duty (Drew \& Martin, 2021). As a result, police officers are likely to avoid or suppress negative emotions that are an expected response to the stress and trauma of their work, often coping with these emotions by abusing alcohol or other drugs, expressing outbursts of anger or committing physical violence, or engaging in other risky and destructive behaviors (Karaffa \& Tochkov, 2013; Pasciak \& Kelley, 2013; Rees \& Smith, 2008).

Previous research (Vogel et al., 2007) has proposed a causal pathway that discourages individuals who are experiencing psychological distress from seeking out support for mental health problems (Figure 1). The first step in this pathway is negative messages - transmitted by police culture and society more generally - about the desirability or social acceptability of seeking help for mental health challenges, which Vogel et al. (2007) refer to as "public stigma". These external beliefs about mental health help-seeking in others can become internalized as "self-stigma", a related but distinct construct that reflects one's personally held beliefs about the social unacceptability for that individual to seek out mental health support, and the perceived negative impact on one's self-worth or self-esteem. This stigma about seeking help subsequently is thought to negatively affect specific attitudes about seeking help; for example, is help-seeking good or bad, or will it be helpful or harmful? Finally, negative attitudes about seeking 
psychological help are proposed to directly influence one's intended utilization of resources for mental health support, and actual help-seeking behavior. <Insert Figure 1 about here $>$

This theoretical model, in which the association between perceived public stigma and reduced help-seeking is mediated by self-stigma and negative help-seeking attitudes, was previously validated in a large sample of undergraduates (Vogel et al., 2007), clearly a very different population from law enforcement professionals. The value that police culture places on emotional control, independence, and traditional notions of masculinity (Karaffa \& Koch, 2016) contributes to high levels of mental health stigma (Drew \& Martin, 2021; Haugen et al., 2017; Soomro \& Yanos, 2019) and generally negative help-seeking attitudes (Karaffa \& Tochkov, 2013). In a sample of 248 sworn police officers in Texas and Oklahoma, Karaffa \& Koch (Karaffa \& Koch, 2016) partially replicated the findings of Vogel et al. (2007) in demonstrating that self-stigma fully mediated the relationship between perceived public stigma and negative treatment-seeking attitudes. The implication of this study is that interventions that target stigma around mental help-seeking may improve police officers' attitudes about seeking help, but the researchers did not investigate the relationship between help-seeking attitudes and help-seeking intentions or behavior. Previous studies in non-police populations have identified associations between help-seeking attitudes and prior utilization of mental health support (Hammer et al., 2018) or future intentions to seek out mental health support (Li et al., 2014). Similar relationships have not, to our knowledge, been demonstrated in a law enforcement population, although there is a widespread belief among the profession that stigma is a significant barrier to help-seeking (Drew \& Martin, 2021).

The primary goal of this study was to validate a hypothesized path model of mental health help-seeking intentions (Vogel et al., 2007) in a law enforcement population. By 
replicating previous work demonstrating the importance of stigma in moderating police employees' attitudes about help-seeking (Karaffa \& Koch, 2016), and further identifying a link between help-seeking attitudes and intentions to seek help in the future, we hope to identify proximal targets for interventions that seek to increase treatment utilization and improve mental health outcomes among police employees. We tested this model in a sample of 259 police employees across two city departments and one university police department in a metropolitan Midwestern U.S. city. We sought to increase the generalizability of previous research with a more representative sample than that of Karaffa et al. (2016), in which over half of the sample represented sergeants or more senior leadership. In addition, we included civilian as well as sworn employees in our survey. Civilians working in law enforcement agencies experience high rates of organizational stress and secondary trauma, but this group is often overlooked in research on mental health in police organizations. Despite limited research in this group, previous studies have identified similar rates and predictors of burnout among civilian and sworn officers (McCarty \& Skogan, 2013), and equivalent (Carleton et al., 2017) or even higher rates of positive anxiety and depression screens for civilians compared to their sworn counterparts in the same agency (Lentz et al., 2020).

A secondary goal of the current study was to investigate potential moderators that may influence mental health help-seeking. The inclusion of both civilian and commissioned staff allowed us to test this factor as a potential moderator, in addition to demographic characteristics including gender and years of police service. We assessed psychological distress (a composite measure of anxiety, depression, and perceived stress) and tested whether these current mental health symptoms influenced stigma, help-seeking attitudes, or the intention to seek help. Additionally, we tested the impact on this pathway of previous participation in an 8-week 
mindfulness training program, which was offered to sworn staff from these agencies as part of previous research with these agencies. These moderator analyses can help identify individual differences that influence the pathway linking stigma, attitudes, and help-seeking intentions, which may facilitate the development of tailored interventions designed to promote help-seeking behavior among police employees.

\section{Methods}

\section{Pre-registration}

Hypotheses and data collection and analysis plans were pre-registered prior to data collection in May 2020 on the Open Science Framework (https://osf.io/zyk9d). The overarching questions addressed in this pre-registration were: 1) What factors influence police employees' attitudes, intentions, and behaviors related to seeking mental health help? and 2) What is the impact of mandatory, agency-wide mental health/wellness checks on shifting these attitudes, intentions, and behaviors? One of the participating agencies planned to implement these mandatory wellness checks in mid-2020, but funding cuts led to the elimination of wellness checks from that department's budget. As such, the focus of this paper is on pre-registered and exploratory analyses utilizing data from a single time point. Other changes to our pre-registration are indicated in relevant sections below. The pre-registered hypotheses we tested were:

Hypothesis 1 (mental health help-seeking stigma and help-seeking attitudes)

- H1a: Higher levels of perceived stigma and self-stigma of mental health help-seeking will be correlated with more negative attitudes toward help-seeking

- H1b: Self-stigma of seeking help will mediate the relationship between perceived stigma of seeking help and attitudes toward seeking help 
- H1c: Previous participation in an 8-week mindfulness training program will be associated with reduced perceived and self-stigma and more positive attitudes toward help-seeking

- H1d: Higher levels of anxiety and depression symptoms will be associated with increased perceived and self-stigma and more negative attitudes toward help-seeking Hypothesis 2 (mental health help-seeking attitudes and past/future help-seeking)

- H2a: More positive attitudes toward mental health help-seeking will be correlated with greater utilization of mental health resources over the previous 12 months

- H2b: More positive attitudes toward mental health help-seeking will be correlated with a higher stated willingness to utilize mental health resources in the future

- H2c: Previous participation in an 8-week mindfulness training program will be associated with increased past 12-month utilization of mental health resources and a higher stated willingness to utilize mental health resources in the future

- H2d: Higher levels of anxiety and depression symptoms will be associated with a lower stated willingness to utilize mental health resources in the future

\section{Participants and Recruitment}

We partnered with two city police departments and one university police department in a mid-sized, Midwestern U.S. metropolitan area on this survey study (our pre-registration indicated we would partner with two agencies, but we added a third to increase our sample size and generalizability). All civilian and sworn employees across all job ranks and positions were invited to take part in this research. Before distributing the survey, we collected feedback on survey questions, methods, and participants from management and union representatives of one partnering agency (including both sworn and civilian staff). Beginning on May 4, 2020, the study was promoted at daily briefings for all shift changes. Up to three emails advertising the study 
were distributed to all employees over the following two weeks, one of which included a link to a recruitment video recorded by the study investigator. The recruitment email included a link to an anonymous Qualtrics survey, the first page of which included a description of study procedures and the opportunity for participants to provide informed consent. Notably, this survey was administered as each of these agencies was adapting to rapidly changing operational conditions in the early months of the COVID-19 pandemic. Additionally, the end of data collection took place in the days following the murder of George Floyd by Minneapolis police officer Derek Chauvin on May 25, 2020. The majority of data collection was completed by that date and only 7/259 responses were obtained after May 25.

\section{Data Collection}

Anonymous survey data were collected using the Qualtrics platform. Based on best practices for anonymous data collection in longitudinal research (Audette et al., 2020), we asked participants to generate a unique alphanumeric code that would allow us to link future survey responses to this initial response. To further protect participants' identity and encourage honest responding, information on demographics and job characteristics were collected using sufficiently broad bins to preclude identification of individual respondents. Participants were allowed and encouraged (in recruitment emails and advertisements at briefings) to complete the survey during work time, but were not compensated for their participation. The median time to complete all survey questions was 25 minutes. The measures obtained from participants (in order) included:

1. Demographics and job information. This in-house measure asked participants to report their employer, civilian/sworn status, job position, tenure working in law enforcement, gender identity, age range, race, ethnicity, and relationship status. 


\section{Patient-Reported Outcomes Measurement Information System, 29-item version}

(PROMIS-29; Hays et al., 2018), a brief assessment of anxiety, depression, fatigue, sleep disturbances, ability to participate in social roles and activities, physical function, pain interference, and pain intensity. Primary analyses included standardized t-scores for anxiety and depression subscales.

3. Perceived Stress Scale, 10-item version (PSS-10; Cohen \& Williamson, 1988), assessing the degree to which life events are appraised as stressful. Analyses used total scores.

4. Perceived Stigma and Barriers to Care for Psychological Problems (Britt \& Cox, 2008), which contains two factors related to perceived public stigma of seeking help for mental health problems (i.e., "what would others think about me if I sought help?”) and other barriers to care. Wording for some items from this scale, originally developed for use in military populations, was modified to be relevant to police employees. An additional item of high relevance for police employees was also incorporated ("I would worry about my personal problems being part of my work records"). The adapted version contained 5 items for barriers to care and 7 items for perceived public stigma. Analyses focused on total scores for the perceived public stigma items.

5. Self-Stigma of Seeking Help Scale (Vogel et al., 2006), a 10-item scale assessing beliefs about what it would mean to seek professional help for mental health concerns (i.e., “what would I think about myself if I sought help?”). Self-stigma can be conceptualized as the internalization of perceived public stigma of seeking help (Vogel et al., 2007).

6. Mental Help Seeking Attitudes Scale (Hammer et al., 2018), a 9-item scale that asks participants to indicate their attitudes about seeking help from a mental health professional. Bipolar pairs of words are presented in response to the prompt: "If I had a 
mental health concern, seeking help from a mental health professional would be ...”, with pairs such as "Useless / Useful", "Good / Bad", and "Disempowering / Empowering". This scale measures one's general perceptions (positive or negative) of mental health counseling (Vogel et al., 2007). Bipolar responses were converted to a 1-7 range and sum scores were used for analysis, with higher scores indicating more positive attitudes.

7. Past and future utilization of mental health resources. This in-house measure was informed by previous research on mental health resources in Canadian public safety personnel (Carleton et al., 2020), with additional items added based on input from law enforcement partners and the investigator's previous research experience. Fourteen potential resources for mental health support were listed, and participants were asked whether they had used each resource in the past 12 months, longer than 12 months ago, or never at all. For any resources used in the past, they were also asked to rate how helpful they found each resource. Finally, the same 14 resources were presented and participants were asked to indicate their willingness to utilize this resource in the future if they needed mental health support ("would not consider"/“might consider"/“would definitely consider using this resource to support my mental health"). For data analysis, we summed the total number of resources utilized in the past 12 months and the total number of resources participants would maybe or definitely utilize in the future.

8. Agency culture and climate related to mental health. Perceptions of the agency culture and climate related to mental health was assessed using 4 Likert items with response options from "strongly disagree" to "strongly agree": "My department cares about the mental health of its employees"; "My department is doing enough to support the mental health of its employees"; "My department has policies and practices in place that protect 
employees who seek out help with mental health concerns"; "I am aware of the mental health resources that are available to me from my department". An additional open-ended question asked participants, "What can your department start doing to better support employee mental health?”. These items were primarily collected as feedback for participating agencies and data were not used in the current analysis.

9. Previous mindfulness training. Participants were asked whether they participated in an 8week mindfulness training class previously provided to some sworn personnel through our research center (Grupe, Smith, et al., 2021; Grupe, Stoller, et al., 2021). For participants who took part in this training, follow-up questions asked how frequently they had engaged in mindfulness practices over the past month and whether they had found benefit in these practices.

10. Questionnaires assessing impact and experiences related to COVID-19. We administered validated surveys to assess COVID-19 fear, direct experiences, and personal impact (Conway et al., 2020). Additional questionnaires were developed for this study to assess 1) police-specific experiences of COVID-19 (i.e., impact on the work experience), 2) work-related stress related to different aspects of the COVID-19 epidemic, and 3) coping strategies used to address COVID-related stress. Items were derived largely from surveys administered to healthcare workers during a coronavirus outbreak in Saudi Arabia in spring 2014 (Khalid et al., 2016) and from the Brief COPE (Carver, 1997).

\section{Data Analysis}

Statistical analysis was conducted in two stages using RStudio (version 1.2.5042;

RStudio Team, 2020) in the R programming environment (version 3.6.3; R Core Team, 2020).

Figures were created using the ggplot2() (Wickham, 2016) and ggpubr() (Kassambara, 2020) 
libraries. We first conducted linear regression analyses to test pre-registered hypotheses concerning factors associated with individual differences in help-seeking attitudes (Hypothesis 1) and past or future (intended) utilization of resources to address mental health concerns (Hypothesis 2). Our pre-registration proposed using the lmerTest() library to account for the nonindependence of participants within each agency, but due to non-convergence errors (likely due to the small number of agencies), we instead conducted linear regression analyses using the $\operatorname{lm}()$ library. While the specific variables differed across analyses, all models took the same essential form, namely:

$$
\operatorname{lm}(\mathrm{DV} \sim \mathrm{IV}+\text { agency }+ \text { civilianCommissioned }+ \text { gender }+ \text { yearsExperience })
$$

where 'agency' was a categorical variable indicating 1 of 3 law enforcement agencies; 'civilianCommissioned' was a categorical variable based on job classification; 'gender' was a categorical variable with possible values of male, female, or non-binary; and 'yearsExperience' was a factor with levels of $0-5,5-9,10-14,15-19$, and $20+$ years of experience working in a police agency. Some models included a moderator variable of previous mindfulness training or psychological distress symptoms (operationalized as the standardized sum score of scores on the Perceived Stress Scale and PROMIS anxiety and depression scores) in addition to the IV.

For regression analyses, univariate outliers were defined as scores on the measure of interest greater than $3 \mathrm{SD}$ from the mean and distinct from the distribution. Model outliers were defined as data points with Cook's D values greater than a cutoff threshold of 4/df that were disconnected from the distribution. Univariate and multivariate outliers were excluded from analyses on a casewise basis.

In addition to running separate regression models for individual dependent variables, we used the lavaan() library (Rosseel, 2011) to conduct a path analysis empirically testing the fit of a 
model linking together perceived stigma of help-seeking, self-stigma of help-seeking, helpseeking attitudes, and help-seeking intentions (Lin, 2021; Figure 1). We initially included the same covariates from regression analyses as exogenous variables (civilian/commissioned status, gender, and years of work experience), but removed agency from the model as it had no impact on any endogenous variables (all $p \mathrm{~s}>0.4$ ). To test hypothesized moderators of this path leading to help-seeking intentions, we constructed a model with additional exogenous variables corresponding to prior mindfulness training and psychological distress symptoms.

\section{Results}

Table 1 includes information on sample characteristics, including demographics and work information. Across the three agencies, 276 individuals clicked on the Qualtrics link, 259 provided informed consent and at least some survey data, and 249 completed all surveys. The 259 individuals who answered questions about work information included 195 sworn and 64 civilian staff. The number of individuals providing informed consent was approximately $20-30 \%$ of employees from each of these agencies, which generally reflects the ratio of civilian/sworn staff in these departments. The two groups had similar age distributions, and both groups were overwhelmingly white (94\% of participants who reported race) and non-Hispanic (93\%). Civilian staff had less experience in law enforcement than sworn staff ( $62 \%$ of civilian staff had fewer than 10 years of experience, compared to $38 \%$ of sworn staff), and $69 \%$ of civilian staff reported female gender compared to $40 \%$ of sworn staff. <Insert Table 1 about here $>$

Table 2 contains descriptive information on each of the self-report measures included in analysis. Relative to sworn staff, civilian staff reported significantly greater levels of perceived stress $(t(250)=2.27, p=0.024,95 \% \mathrm{CI}[0.28,3.89])$, anxiety $(t(250)=3.66, p<0.001,95 \% \mathrm{CI}$ $[1.78,5.87])$, and depression $(t(250)=3.95, p<0.001,95 \% \mathrm{CI}[2.26,6.75])$. Group differences 
for anxiety and depression (but not perceived stress) remained significant when controlling for gender and years of work experience. The two groups did not differ in perceived stigma, selfstigma, or mental help-seeking attitudes (all $|t \mathrm{~s}|<1.4$, all $p \mathrm{~s}>0.1$ ). Sworn staff reported utilizing a greater number of resources for mental health support in their lifetime $(t(248)=2.07, p=0.04$, $95 \% \mathrm{CI}[0.04,1.53])$, and a trend toward greater utilization in the past 12 months $(t(248)=1.79$, $p=0.07,95 \%$ CI $[-0.05,1.16])$ and greater intended future utilization $(t(247)=1.71, p=0.09$, 95\% CI $[-0.09,1.30]) .<$ Insert Table 2 about here $>$

\section{Mental Health Stigma and Help-Seeking Attitudes}

Controlling for agency, civilian/commissioned status, gender, and years of experience, greater perceived public stigma of mental health help-seeking was associated with more negative help-seeking attitudes $\left(r_{\text {partial }}(249)=-0.36,95 \%\right.$ CI $[-0.46,-0.25], p<0.001$; Figure 2a). We observed an even more robust relationship between self-stigma and attitudes toward mental health help-seeking $\left(r_{\text {partial }}(249)=-0.63,95 \%\right.$ CI $[-0.70,-0.55], p<0.001$; Figure 2b). The relationship between help-seeking attitudes and self-stigma was stronger than that for perceived public stigma, as tested using the cocor() library (Diedenhofen \& Musch, 2015); William's $t(248)=6.20, p<0.001)$. These correlations were of comparable magnitude to those observed using different measures of stigma and help-seeking attitudes in a prior study of 248 sworn officers in Texas and Oklahoma (Karaffa \& Koch, 2016). Also consistent with findings from this prior study, mediation analysis using lavaan() demonstrated that the relationship between perceived public stigma and help-seeking attitudes was fully mediated by self-stigma (total effect estimate $=-0.053, p<0.001,95 \%$ CI $[-0.069,-0.037]$; direct effect estimate $=0.00, p=0.99$, $95 \%$ CI $[-0.017,0.018] ;$ indirect effect estimate $=-0.053, p<0.001,95 \%$ CI $[-0.070,-0.040]$; Figure 2c). $<$ Insert Figure 2 about here $>$ 
We next tested the hypothesis that prior mindfulness training would be associated with reduced stigma and more positive attitudes related to mental health help-seeking. Among 184 sworn staff with complete data, $58(31.5 \%)$ reported having previously participated in an 8 -week mindfulness training program. Controlling for agency, age, and years of police service, prior mindfulness training was associated with a trend-level reduction in perceived public stigma $(t(174)=-1.70, p=0.09, b=-2.16,95 \%$ CI $[-4.67,0.35])$ but not self-stigma $(t(174)=-1.06, p=$ $0.29, b=-1.34,95 \% \mathrm{CI}[-3.84,1.16])$, and a trend toward more positive help-seeking attitudes $(t(174)=1.68, p=0.10, b=0.31,95 \%$ CI $[-0.06,0.67])$.

We also tested the hypothesis that individuals with greater symptoms of psychological distress (a composite index of anxiety, depression, and perceived stress) would report greater help-seeking stigma and more negative help-seeking attitudes. Consistent with hypotheses, greater psychological distress was associated with greater perceived stigma $\left(r_{\text {partial }}(250)=0.36\right.$, $95 \%$ CI $[0.25,0.47], p<0.001)$, greater self-stigma $\left(r_{\text {partial }}(250)=0.24,95 \% \mathrm{CI}[0.12,0.35], p<\right.$ $0.001)$, and more negative attitudes related to mental health help-seeking $\left(r_{\text {partial }}(249)=-0.20\right.$, 95\% CI [-0.32, -0.08], $p=0.001)$, controlling for agency, civilian/commissioned status, age, and years of police service.

\section{Help-Seeking Attitudes and Intended Help-Seeking Behavior}

We created a novel questionnaire for this study that listed 14 different resources that police employees might seek out for mental health support. On average, participants reported utilizing 6.3 of these resources in their lifetime (range $=0-13$ ) and 4.1. of these resources over the past 12 months (range $=0-10$ ). Sworn personnel reported utilizing slightly more resources than civilian staff over the past 12 months $(t(109.3=1.74, p=0.08, b=0.56,95 \% \mathrm{CI}[-0.08$, 1.19]) and overall $(t(112.3=2.04, p=0.04, b=0.79,95 \% \mathrm{CI}[0.02,1.55])$. Across all 
respondents, the most frequently utilized lifetime resources were "support from a spouse, partner, or family member" ( $87.3 \%$ of respondents), "support from a friend outside of law enforcement" (81.7\%), and "support from a friend within law enforcement" (78.9\%), followed by "mindfulness or meditation" (68.1\%) and "critical incident stress debriefing" (64.9\%; Table 3). The least utilized resources by far were "crisis hotline" (2.0\%) and "police chaplain" (1.6\%), the latter of which was not an available resource for participants in these agencies.

We also asked participants to indicate which of these resources they would maybe or definitely seek out if they needed mental health support in the future. One participant was removed from these analyses for being an outlier, as they endorsed 0/14 resources (the next lowest value was 4/14). The top five resources participants would seek out remained unchanged from the most frequently used past resources (Table 3). Notably, whereas "support from a supervisor or police leadership" was the 6th most frequently utilized resource in the past (53.8\%), this resource fell to $\# 10$ for resources likely to be utilized in the future (30.7\%). $<$ Insert

\section{Table 3 about here $>$}

To measure previous and future (intended) mental health help-seeking behavior, we tallied the number of distinct resources participants indicated they had utilized in the past 12 months, and those they "might consider" or "would definitely consider" seeking out if they needed mental health support in the future. Controlling for agency, civilian/commissioned status, age, and years of police service, more positive mental health help-seeking attitudes were associated with greater utilization of mental health support in the past 12 months $\left(r_{\text {partial }}(248)=\right.$ $0.28,95 \%$ CI [0.16, 0.39], $p<0.001$; Figure 3a). We observed a more robust relationship between help-seeking attitudes and future intentions to seek support for mental health needs 
$\left(r_{\text {partial }}(247)=0.45,95 \%\right.$ CI $[0.35,0.55], p<0.001 ;$ difference between correlations: William's $t(246)=2.48, p=0.01 ;$ Figure 3b). $<$ Insert Figure 3 about here $>$

We also tested the role of prior mindfulness training and psychological distress symptoms on past mental health help-seeking behavior and future help-seeking intentions. Controlling for agency, age, and years of police service, prior mindfulness training was associated with greater utilization of mental health resources over the past 12 months $(t(173)=2.50, p=0.01, b=0.84$, $95 \% \mathrm{CI}[0.18,1.50])$ and a trend toward greater intended future utilization $(t(173)=1.63, p=$ $0.10, b=0.63,95 \% \mathrm{CI}[-0.13,1.40])$. Controlling for agency, civilian/commissioned status, age, and years of police service, greater psychological distress was associated with greater utilization of mental health resources over the past 12 months $\left(r_{\text {partial }}(248)=0.13,95 \%\right.$ CI $[0.01,0.26, p=$ $0.03)$, but lower intended future utilization of these resources $\left(r_{\text {partial }}(248)=-0.16,95 \%\right.$ CI $[-0.28$, $-0.04], p=0.01)$.

\section{Path Analysis of Mental Health Help-Seeking Stigma, Attitudes, and Intentions}

Using lavaan(), we modeled the path analysis depicted in Figure 1 in which perceived public stigma predicts self-stigma, which in turn predicts help-seeking attitudes, which in turn predicts intended mental health help-seeking. Exogenous variables that were allowed to predict all endogenous variables were civilian/commissioned status, age, and years of police service. We did not include police agency as an exogenous variable as it was weakly associated with all endogenous variables (all $p \mathrm{~s}>0.4)$. Fit indices suggested this model fit the data well $\left(\chi^{2}(3)=\right.$ $6.420, p=0.093$; comparative fit index $(\mathrm{CFI})=0.988$; Tucker-Lewis Index $(\mathrm{TLI})=0.927$; root mean square error of approximation (RMSEA) $=0.068, p=0.268$; Akaike Information Criterion $(\mathrm{AIC})=5059)$. Because this model was over-identified (i.e., not all possible paths were modeled), we used the modindices() function to obtain the modification index for each omitted 
path, which suggested adding a direct path from perceived public stigma to intended mental health help-seeking. Adding this direct path yielded a model with improved fit indices $\left(\chi^{2}(2)=\right.$ $0.086, p=0.958 ; \mathrm{CFI}=1.000 ; \mathrm{TLI}=1.000 ; \mathrm{RMSEA}=0.000, p=0.977 ; \mathrm{AIC}=5054)$ and for which all fit indices were low (all indices $<0.07$ ). In addition to significant paths between the endogenous variables in this model, there was a significant effect of civilian/commissioned status on intended mental health help-seeking, such that commissioned staff indicated greater intended future use of mental health resources $(Z=2.30, b=0.76, p=0.02$; Figure 4a). $<$ Insert Figure 4 about here $>$

We also tested a model that included distress symptoms and prior mindfulness training to test moderation of this pathway by these two factors. This model again provided an excellent fit to the data $\left(\chi^{2}(8)=4.563, p=0.803 ; \mathrm{CFI}=1.000 ; \mathrm{TLI}=1.000 ; \mathrm{RMSEA}=0.000, p=0.957\right.$; $\mathrm{AIC}=5021$; Figure 4b). Examination of individual pathways for hypothesized moderators showed significant direct effects on perceived public stigma for distress symptoms $(Z=6.07, b=$ $3.25, p<0.001)$ and prior mindfulness training $(Z=-2.21, b=-2.52, p=0.03)$. In other words, psychological distress indirectly led to lower intended help-seeking through an increase in perceived public stigma, whereas prior mindfulness training indirectly led to higher intended help-seeking through a decrease in perceived public stigma. The inclusion of these moderators also revealed a significant effect of civilian/commissioned status on perceived public stigma, with greater stigma for commissioned vs. civilian employees $(Z=3.06, b=3.51, p=0.002)$.

\section{Discussion}

Results of this cross-sectional survey study in 259 sworn and civilian police employees provide strong support for a pathway progressing from perceived public stigma around mental health help-seeking to internalized self-stigma, which leads to negative help-seeking attitudes, 
and in turn to reduced intentions to seek out mental health support. Symptoms of psychological distress were associated with higher levels of perceived public stigma, indirectly contributing to decreased help-seeking intentions. On the other hand, prior participation in an 8-week mindfulness training program was associated with lower levels of perceived public stigma, indirectly contributing to increased help-seeking intentions.

These findings underscore the importance of perceived public stigma -- in other words, police employees' beliefs about what others will think of them if they were to seek help for mental health concerns -- for mental health help-seeking. A valuable concept to consider in this regard is "pluralistic ignorance", which occurs when the majority of individual group members privately reject a norm even as they believe the majority of others in the group embrace this norm (Katz \& Allport, 1931). Previous studies have demonstrated the presence of pluralistic ignorance around mental health help-seeking in police officers, finding that police officers underestimated the extent to which their coworkers would want to seek help for mental health concerns (Karaffa \& Koch, 2016), and in general believed that others were less interested in seeking help than they were themselves (Karaffa \& Tochkov, 2013). On the other hand, the strongest predictor of positive help-seeking attitudes was the perception that one's coworkers were interested in mental health support (Karaffa \& Tochkov, 2013). Given a context in which there is strong pressure to identify with other group members, and in which conversations about mental health are discouraged (at least implicitly), there may be a failure among police employees to recognize that others' behaviors and attitudes around mental help-seeking reflect a desire to fit in with the group, as opposed to personally held beliefs and values. By encouraging greater dialogue around help-seeking, and highlighting discrepancies between perceptions and realities of coworkers' attitudes about help-seeking, police organizations may be able to correct 
erroneous beliefs of "what everybody else thinks" and consequently encourage greater helpseeking behavior through reductions in perceived public stigma (Karaffa \& Koch, 2016).

Notably, we do not have data that speak to actual levels of public stigma of help-seeking among these departments, and it may be the case that perceptions about high levels of public stigma are in fact accurate. As discussed in Vogel et al. (2007), moving the needle on public stigma is challenging because it requires widespread cultural changes, perhaps made more difficult by a police culture that values independence and stoicism, and that views emotional difficulties as a sign of weakness that can compromise one's professional competency (Drew \& Martin, 2021; Karaffa \& Koch, 2016; Karaffa \& Tochkov, 2013; Tuckey et al., 2012). Even if police employees accurately perceive help-seeking stigma among their coworkers to be high, however, all is not lost. We found that the negative impact of perceived public stigma on attitudes toward help-seeking was fully mediated by self-stigma, consistent with previous studies in undergraduate (Vogel et al., 2007) and police samples (Karaffa \& Koch, 2016). This result suggests that changes in widespread cultural beliefs about mental help-seeking may not be necessary to promote help-seeking behavior so long as interventions can effectively combat selfstigma around mental help-seeking.

We are unaware of any interventions that have explicitly targeted self-stigma about helpseeking in police employees. A report commissioned as part of the 2017 Law Enforcement Mental Health and Wellness Act (Spence et al., 2019) suggested that regular mental health check-ins with mental health professionals are increasingly being used as a means of reducing help-seeking stigma, but also noted the total lack of empirical evidence for the effects of these check-ins. Although a one-time mental health check-in seems unlikely to serve as an effective intervention by itself for a police officer struggling with mental illness, it could help destigmatize 
the help-seeking experience and encourage additional help-seeking behavior. Making these check-ins mandatory for all employees is likely to be an important aspect for destigmatizing help-seeking, as all employees are talking to a counselor, not just those who have "a problem" (Spence et al., 2019).

Stigma about help-seeking likely reflects a more fundamental stigma about what it means to experience strong negative emotions that police employees perceive may compromise their competency or efficacy or that they believe their coworkers are not experiencing (Tuckey et al., 2012). A "foundational step" in increasing uptake of mental health services, argue Drew \& Martin (2021), is normalizing the strong negative emotions that naturally arise from working in an environment in which police officers are regularly exposed to significant trauma and human suffering. Acknowledging, noticing, and talking about the emotional responses that are to-be expected in this work -- rather than avoiding or suppressing these emotions to project a facade of strength and control -- may provide a foundation for facilitating help-seeking when the consequences of exposure to occupational stress and trauma are too great for individuals to bear alone. Engaging in these conversations in a group setting allows individuals to recognize they are not the only ones who experience these emotions, and that perceived public stigma about discussing emotional difficulties is largely a false perception that everybody goes along with to be part of the group.

In support of this strategy to reduce stigma, we found that previous participation in an 8week, group-based mindfulness training was a significant moderator of the path proceeding from perceived public stigma to help-seeking intentions. Previous mindfulness training participants also reported seeking out significantly more sources of support for mental health difficulties over the past 12 months compared to non-participants. Among other elements, this training program 
supports participants in developing greater awareness of internal sensations, emotions, and thoughts; fostering a curious and accepting stance toward these experiences, rather than ignoring or suppressing them; and talking with other group members about these experiences and how they affect participants personally and professionally. Because relationships between prior mindfulness training, reduced stigma, and increased help-seeking are correlational, it is possible that individuals who voluntarily signed up for this training had lower levels of stigma or more positive attitudes toward help-seeking prior to mindfulness training. Future research on mindfulness-based interventions for law enforcement should include as outcomes measures of help-seeking stigma, attitudes, and subsequent help-seeking behavior to establish a causal role of mindfulness for promoting mental health help-seeking.

Symptoms of psychological distress had the opposite effect on this pathway as prior mindfulness training, as they were associated with greater perceived stigma and reduced intentions to seek help in the future. These associations were observed despite a positive association between distress symptoms and previous help-seeking, suggesting that participants' past help-seeking experiences may have been ineffective and dissuading them from future helpseeking. Elevated stigma among those with high levels of anxiety, depression, and perceived stress presents a paradox for getting employees into treatment, and suggests that mental health interventions may need to be framed in terms of skill-building or as addressing less stigmatized needs (e.g., improving sleep or strengthening relationships) in order to engage those police personnel experiencing high levels of both stigma and mental health symptoms. Some of the resources in which participants reported the greatest interest were not mental health interventions per se, but support from family, friends, and peers. Providing members of these existing support 
networks with resources or knowledge about supporting individuals with mental health needs could be a valuable intervention strategy that skirts the barrier of help-seeking stigma.

A notable strength of this study was the inclusion of civilian police employees, who made up $25 \%$ of the total sample. Civilian staff are frequently overlooked in research on law enforcement mental health, perhaps due to an assumption that patrol officers and other sworn personnel have greater mental health needs due to the nature of their work. Civilian staff, however, experience rates of burnout, anxiety, and depression that are comparable or even greater than commissioned personnel (Carleton et al., 2017; Lentz et al., 2020; McCarty \& Skogan, 2013). Indeed, civilian staff in the current study also reported significantly greater levels of perceived stress, anxiety, and depression than sworn staff. Although civilian staff endorsed fewer resources they would be likely to seek out in the future (mean 5.7 vs. 6.5), this comparison is complicated by the fact that some resources available to sworn staff are not available to civilians in these agencies (e.g., peer support, critical incident debriefing). In the path analysis that simultaneously controlled for individual differences in distress symptoms, civilian vs. sworn staff endorsed lower levels of perceived public stigma which contributed indirectly to increased intended help-seeking. This snapshot of group differences highlights the importance of including civilian as well as sworn staff in future research on law enforcement mental health, which will help shed light on the unique and shared needs of all members of the organization.

\section{Limitations and Implications for Policy and Future Research}

Despite the inclusion of multiple police departments and civilian as well as sworn staff, this study is limited in its generalizability due to the constrained geographical representation of the sample and the over-representation of employees from a single agency (over $80 \%$ of responses were from the Madison Police Department, by far the largest agency among the 3 
included). Further, while all employees were invited to participate, the sample size of 259 only represents about $30 \%$ of employees across these agencies, and our results may be biased by selfselection. These concerns about generalizability may be lessened somewhat by the observation that relationships between perceived stigma, self-stigma, and help-seeking attitudes were nearly identical in magnitude to a previous study from a different geographical region with sworn staff only (Karaffa \& Koch, 2016). The relative racial and ethnic homogeneity, reflective of the personnel of the police agencies with which we collaborated, limits the extent to which we can generalize results to non-white police employees.

Another limitation is the use of a novel measure of intended help-seeking that has not been validated, one that quantifies the number of resources participants would seek out rather than their likelihood of seeking help of any kind. We made the decision to include this novel measure rather than a more generic yet validated help-seeking scale (e.g., Cash et al., 1975) so we could obtain specific, qualitative information on the types of resources that employees have used or would be interested in utilizing. This decision increases the external validity of results but limits the ability to draw a direct comparison to other studies examining help-seeking intentions (although this concern may be mitigated by the consistency between our results and prior work linking stigma, help-seeking attitudes, and willingness to seek counseling in an undergraduate sample; Thornicroft et al., 2016; Vogel et al., 2007).

We close with two implications of this work for police organizations specifically and for society more broadly. First is the importance of considering multi-level interventions to increase mental health help-seeking among police employees. Efforts at the individual level to reduce self-stigma and raise awareness of the existence and impact of pluralistic ignorance cannot be done at the exclusion of addressing organizational and cultural messages about the personal and 
professional consequences of acknowledging difficulties and seeking help. Interventions to reduce mental health stigma among the general public are most effective at changing attitudes when they are based on social contact and first-person narratives and supported by a long-term commitment (Thornicroft et al., 2016). Management and direct supervisors thus have an important role in changing organizational culture by providing clear, consistent, and personal communication that normalizes mental health difficulties, invites conversations about mental health, and makes explicit the protections in place for employees who seek help.

More broadly, we argue that interventions that promote help-seeking behavior and benefit police mental health will be of indirect benefit for community mental health, well-being, and physical safety, and should be considered as part of larger police reform efforts. As the author and therapist Resmaa Menakem wrote, "It's hard to keep the peace when your own body is constricted, unsettled, stressed, and traumatized" (Menakem, 2017, p. 125). It is critical for the safety and well-being of all that we identify novel strategies to combat help-seeking stigma, promote help-seeking behavior for all who need it in an individualized and culturally appropriate manner, and help police professionals address the unresolved and unmetabolized trauma that too often results in tragedy. This work affects community members and police practitioners alike, and identifying creative solutions necessitates collaboration among these groups to ensure that these solutions are of mutual benefit. 


\section{References}

Audette, L. M., Hammond, M. S., \& Rochester, N. K. (2020). Methodological Issues with Coding Participants in Anonymous Psychological Longitudinal Studies. Educational and Psychological Measurement, 80(1), 163-185. https://doi.org/10.1177/0013164419843576

Britt, T. W., Greene-Shortridge, T. M., Brink, S., Nguyen, Q. B., Rath, J., Cox, A. L., Hoge, C. W., \& Castro, C. A. (2008). Perceived stigma and barriers to care for psychological treatment: Implications for reactions to stressors in different contexts. Journal of Social and Clinical Psychology, 27(4), 317-335. https://doi.org/10.1521/jscp.2008.27.4.317

Carleton, R. N., Afifi, T. O., Turner, S., Taillieu, T., Duranceau, S., LeBouthillier, D. M., Sareen, J., Ricciardelli, R., MacPhee, R. S., Groll, D., Hozempa, K., Brunet, A., Weekes, J. R., Griffiths, C. T., Abrams, K. J., Jones, N. A., Beshai, S., Cramm, H. A., Dobson, K. S., ... Asmundson, G. J. G. (2017). Mental Disorder Symptoms among Public Safety Personnel in Canada. The Canadian Journal of Psychiatry, 63(1), 54-64. https://doi.org/10.1177/0706743717723825

Carleton, R. N., Afifi, T. O., Turner, S., Taillieu, T., Vaughan, A. D., Anderson, G. S., Ricciardelli, R., MacPhee, R. S., Cramm, H. A., Czarnuch, S., Hozempa, K., \& Camp, R. D. (2020). Mental health training, attitudes toward support, and screening positive for mental disorders. Cognitive Behaviour Therapy, 49(1), 55-73.

https://doi.org/10.1080/16506073.2019.1575900

Carver, C. S. (1997). You Want to Measure Coping but Your Protocol's Too Long: Consider the Brief COPE. International Journal of Behavioral Medicine, 4(1), 92-100. https://doi.org/10.1207/s15327558ijbm0401_6 
Cash, T. F., Begley, P. J., McCown, D. A., \& Weise, B. C. (1975). When counselors are heard but not seen: Initial impact of physical attractiveness. Journal of Counseling Psychology, 22(4), 273-279. https://doi.org/10.1037/H0076730

Cohen, S., \& Williamson, G. (1988). Perceived stress in a probability sample of the United States. In The Social Psychology of Health (Vol. 13, pp. 31-67). https://doi.org/10.1111/j.1559-1816.1983.tb02325.x

Conway, L. G., Woodard, S. R., \& Conway, L. G. (2020). Social Psychological Measurements of COVID-19: Coronavirus Perceived Threat, Government Response, Impacts, and Experiences Questionnaires. https://psyarxiv.com/z2x9a/.

Diedenhofen, B., \& Musch, J. (2015). cocor: A Comprehensive Solution for the Statistical Comparison of Correlations. PLOS ONE, 10(4), e0121945. https://doi.org/10.1371/JOURNAL.PONE.0121945

Drew, J. M., \& Martin, S. (2021). A National Study of Police Mental Health in the USA: Stigma, Mental Health and Help-Seeking Behaviors. Journal of Police and Criminal Psychology, 36(2), 295-306. https://doi.org/10.1007/s11896-020-09424-9

Goff, P. A., \& Rau, H. (2020). Predicting Bad Policing: Theorizing Burdensome and Racially Disparate Policing through the Lenses of Social Psychology and Routine Activities. Annals of the American Association of Political and Social Sciences, 687, 67-88. https://doi.org/10.1177/0002716220901349

Grupe, D. W., Smith, C., \& McGehee, C. (2021). Introducing mindfulness training and research into policing: Strategies for successful implementation. In E. Arble \& B. B. Arnetz (Eds.), Interventions, Training, and Technologies for Improved Police Well-Being and Performance. IGI Global. 
Grupe, D. W., Stoller, J. L., Alonso, C., McGehee, C., Smith, C., Mumford, J. A., Rosenkranz, M. A., \& Davidson, R. J. (2021). The Impact of Mindfulness Training on Police Officer Stress, Mental Health, and Salivary Cortisol Levels. Frontiers in Psychology, 12(September). https://doi.org/10.3389/fpsyg.2021.720753

Hammer, J. H., Parent, M. C., \& Spiker, D. A. (2018). Mental help seeking attitudes scale (MHSAS): Development, reliability, validity, and comparison with the ATSPPH-SF and IASMHS-PO. Journal of Counseling Psychology, 65(1), 74-85.

https://doi.org/10.1037/cou0000248

Haugen, P. T., McCrillis, A. M., Smid, G. E., \& Nijdam, M. J. (2017). Mental health stigma and barriers to mental health care for first responders: A systematic review and meta-analysis. Journal of Psychiatric Research, 94, 218-229. https://doi.org/10.1016/j.jpsychires.2017.08.001

Hays, R. D., Spritzer, K. L., Schalet, B. D., \& Cella, D. (2018). PROMIS®-29 v2.0 profile physical and mental health summary scores. Quality of Life Research, 27(7), 1885-1891. https://doi.org/10.1007/s11136-018-1842-3

Karaffa, K. M., \& Koch, J. M. (2016). Stigma, Pluralistic Ignorance, and Attitudes Toward Seeking Mental Health Services Among Police Officers. Criminal Justice and Behavior, 43(6), 759-777. https://doi.org/10.1177/0093854815613103

Karaffa, K. M., \& Tochkov, K. (2013). Attitudes Toward Seeking Mental Health Treatment Among Law Enforcement Officers. Applied Psychology in Criminal Justice, 9(2), 75-99. Kassambara, A. (2020). ggpubr: 'ggplot2'Based Publication Ready Plots. https://rpkgs.datanovia.com/ggpubr/index.html 
Katz, D., \& Allport, F. H. (1931). Introduction. In Students'Attitudes: A Report of the Syracuse University Reaction Study (pp. 1-8). Craftsman Press.

Khalid, I., Khalid, T. J., Qabajah, M. R., Barnard, A. G., \& Qushmaq, I. A. (2016). Healthcare workers emotions, perceived stressors and coping strategies during a MERS-CoV outbreak. Clinical Medicine and Research, 14(1), 7-14. https://doi.org/10.3121/cmr.2016.1303

Lentz, L., Silverstone, P. H., \& Krameddine, Y. I. (2020). High Rates of Mental Health Disorders in Civilian Employees Working in Police Organizations. Frontiers in Psychology, 11(May), 1-7. https://doi.org/10.3389/fpsyg.2020.01031

Li, W., Dorstyn, D. S., \& Denson, L. A. (2014). Psychosocial correlates of college students' help-seeking intention: A meta-analysis. Professional Psychology: Research and Practice, 45(3), 163-170. https://doi.org/10.1037/a0037118

Lin, J. (2021). Introduction to Structural Equation Modeling (SEM) in R. https://stats.idre.ucla.edu/r/seminars/rsem/

Ma, D. S., Correll, J., Wittenbrink, B., Bar-Anan, Y., Sriram, N., \& Nosek, B. A. (2013). When Fatigue Turns Deadly: The Association Between Fatigue and Racial Bias in the Decision to Shoot. Basic and Applied Social Psychology, 35(6), 515-524.

https://doi.org/10.1080/01973533.2013.840630

McCarty, W. P., \& Skogan, W. G. (2013). Job-Related Burnout Among Civilian and Sworn Police Personnel. Police Quarterly, 16(1), 66-84. https://doi.org/10.1177/1098611112457357

Menakem, R. (2017). My Grandmother's Hands: Racialized Trauma and the Pathway to Mending Our Hearts and Bodies. Central Recovery Press. 
Pasciak, A. R., \& Kelley, T. M. (2013). Conformity to traditional gender norms by male police officers exposed to trauma: Implications for critical incident stress debriefing. Applied Psychology in Criminal Justice, 9(2), 137-156.

R Core Team. (2020). R: A Language and Environment for Statistical Computing. https://www.r-project.org/

Rajaratnam, S. M. W., Barger, L. K., Lockley, S. W., Shea, S. A., Wang, W., Landrigan, C. P., O’Brien, C. S., Qadri, S., Sullivan, J. P., Cade, B. E., Epstein, L. J., White, D. P., \& Czeisler, C. A. (2011). Sleep disorders, health, and safety in police officers. JAMA, 306(23), $2567-2578$.

Rees, B., \& Smith, J. (2008). Breaking the Silence: The Traumatic Circle of Policing. International Journal of Police Science \& Management, 10(3), 267-279. https://doi.org/10.1350/ijps.2008.10.3.83

Rosseel, Y. (2011). lavaan: An R Package for Structural Equation Modeling. Journal of Statistical Software, 48. https://doi.org/10.18637/jss.v048.i02

RStudio Team. (2020). RStudio: Integrated Development Environment for R. http://www.rstudio.com/

Soomro, S., \& Yanos, P. T. (2019). Predictors of Mental Health Stigma among Police Officers: The Role of Trauma and PTSD. Journal of Police and Criminal Psychology, 34(2), 175183. https://doi.org/10.1007/s11896-018-9285-X

Spence, D. L., Fox, M., Moore, G. C., Estill, S., \& Comrie, N. E. (2019). Law Enforcement Mental Health and Wellness Act: Report to Congress.

Syed, S., Ashwick, R., Schlosser, M., Jones, R., Rowe, S., \& Billings, J. (2020). Global prevalence and risk factors for mental health problems in police personnel: A systematic 
review and meta-analysis. Occupational and Environmental Medicine, 77, 737-747. https://doi.org/10.1136/oemed-2020-106498

Thornicroft, G., Mehta, N., Clement, S., Evans-Lacko, S., Doherty, M., Rose, D., Koschorke, M., Shidhaye, R., O’Reilly, C., \& Henderson, C. (2016). Evidence for effective interventions to reduce mental-health-related stigma and discrimination. The Lancet, 387(10023), 1123-1132. https://doi.org/10.1016/S0140-6736(15)00298-6

Tuckey, M. R., Winwood, P. C., \& Dollard, M. F. (2012). Psychosocial culture and pathways to psychological injury within policing. Police Practice and Research, 13(3), 224-240. https://doi.org/10.1080/15614263.2011.574072

Vogel, D. L., Wade, N. G., \& Haake, S. (2006). Measuring the self-stigma associated with seeking psychological help. Journal of Counseling Psychology, 53(3), 325-337. https://doi.org/10.1037/0022-0167.53.3.325

Vogel, D. L., Wade, N. G., \& Hackler, A. H. (2007). Perceived public stigma and the willingness to seek counseling: The mediating roles of self-stigma and attitudes toward counseling. Journal of Counseling Psychology, 54(1), 40-50. https://doi.org/10.1037/0022-0167.54.1.40

Weiss, D. S., Brunet, A., Best, S. R., Metzler, T. J., Fagan, J. A., \& Marmar, C. R. (2010). Frequency and severity approaches to indexing exposure to trauma: The Critical Incident History Questionnaire for police officers. Journal of Traumatic Stress, 23(6), 734-743. https://doi.org/10.1002/jts.

Wickham, H. (2016). ggplot2: Elegant Graphics for Data Analysis. Springer-Verlag New York. https://ggplot2.tidyverse.org 


\begin{tabular}{|c|c|c|c|}
\hline \multicolumn{4}{|l|}{ Gender } \\
\hline Female & $47(69 \%)$ & $76(40 \%)$ & $123(48 \%)$ \\
\hline Male & $21(31 \%)$ & $113(60 \%)$ & $134(52 \%)$ \\
\hline \multicolumn{4}{|l|}{ Age range } \\
\hline $18-24$ & $3(4 \%)$ & $11(6 \%)$ & $14(5 \%)$ \\
\hline $25-34$ & $21(31 \%)$ & $55(29 \%)$ & $76(30 \%)$ \\
\hline $35-44$ & $15(22 \%)$ & $62(33 \%)$ & $77(30 \%)$ \\
\hline $45-54$ & $17(25 \%)$ & $53(28 \%)$ & $70(27 \%)$ \\
\hline 55 or older & $11(16 \%)$ & $6(3 \%)$ & $17(7 \%)$ \\
\hline Prefer not to say & $1(1 \%)$ & $2(1 \%)$ & $3(1 \%)$ \\
\hline \multicolumn{4}{|l|}{ Race } \\
\hline Asian/Pacific Islander & $2(3 \%)$ & $2(1 \%)$ & $4(2 \%)$ \\
\hline Black & $0(0 \%)$ & $4(2 \%)$ & $4(2 \%)$ \\
\hline Native American & $0(0 \%)$ & $1(1 \%)$ & $1(0 \%)$ \\
\hline White & $63(93 \%)$ & $170(90 \%)$ & $233(91 \%)$ \\
\hline More than one & $1(1 \%)$ & $5(3 \%)$ & $6(2 \%)$ \\
\hline Prefer not to say & $2(3 \%)$ & $7(4 \%)$ & $9(4 \%)$ \\
\hline \multicolumn{4}{|l|}{ Ethnicity } \\
\hline Hispanic or Latinx & $2(3 \%)$ & $15(8 \%)$ & $17(7 \%)$ \\
\hline Not Hispanic or Latinx & $65(96 \%)$ & $169(89 \%)$ & $234(91 \%)$ \\
\hline Prefer not to say & $1(1 \%)$ & $5(3 \%)$ & $6(2 \%)$ \\
\hline \multicolumn{4}{|l|}{ Years of work experience } \\
\hline $0-4$ & $27(39 \%)$ & $45(24 \%)$ & $72(28 \%)$ \\
\hline $5-9$ & $15(22 \%)$ & $27(14 \%)$ & $42(16 \%)$ \\
\hline $10-14$ & $13(18 \%)$ & $37(20 \%)$ & $50(19 \%)$ \\
\hline $15-19$ & $5(7 \%)$ & $32(17 \%)$ & $37(14 \%)$ \\
\hline 20 or more & $8(12 \%)$ & $47(25 \%)$ & $55(21 \%)$ \\
\hline Prefer not to say & $0(0 \%)$ & $1(0 \%)$ & $1(0 \%)$ \\
\hline \multicolumn{4}{|l|}{ Job classification } \\
\hline Civilian non-supervisor & $57(84 \%)$ & - & - \\
\hline Civilian supervisor & $10(15 \%)$ & - & - \\
\hline Police officer & - & $112(59 \%)$ & - \\
\hline Detective/Investigator & - & $32(17 \%)$ & - \\
\hline Sergeant & - & $20(11 \%)$ & - \\
\hline Management & - & $21(11 \%)$ & - \\
\hline Prefer not to say & $1(1 \%)$ & $4(2 \%)$ & - \\
\hline
\end{tabular}

Table 1. Participant demographics and job characteristics. Percentages may not add up to $100 \%$ due to rounding. 
Civilian employees Sworn employees All employees

\begin{tabular}{|c|c|c|c|}
\hline Perceived Stress Scale & $15.7(7.2)$ & $13.6(6.1)$ & $14.2(6.5)$ \\
\hline PROMIS Depression & $52.5(8.4)$ & $48.0(7.8)$ & $49.1(8.2)$ \\
\hline PROMIS Anxiety & $57.3(7.3)$ & $53.5(7.3)$ & $54.5(7.5)$ \\
\hline Distress Composite & $0.34(0.96)$ & $-0.11(0.82)$ & $0.0(0.9)$ \\
\hline Perceived Stigma & $15.2(7.7)$ & $16.4(7.6)$ & $16.1(7.7)$ \\
\hline Self-Stigma & $22.1(7.6)$ & $23.6(7.6)$ & $23.2(7.6)$ \\
\hline Help-Seeking Attitudes & $5.7(1.1)$ & $5.7(1.1)$ & $5.7(1.1)$ \\
\hline \multicolumn{4}{|l|}{$\begin{array}{l}\text { Number of mental } \\
\text { health resources used }\end{array}$} \\
\hline Lifetime & $5.7(2.7)$ & $6.5(2.6)$ & $6.3(2.7)$ \\
\hline Past 12 months & $3.7(2.3)$ & $4.2(2.1)$ & $4.1(2.2)$ \\
\hline May consider in future & $10.2(2.9)$ & $10.8(2.3)$ & $10.6(2.5)$ \\
\hline
\end{tabular}

Table 2. Descriptive information for self-report questionnaires. All values reflect means (standard deviations in parentheses). 


\begin{tabular}{|c|c|c|c|c|c|c|c|c|}
\hline \multicolumn{5}{|c|}{ Percent who utilized resource in past } & \multicolumn{4}{|c|}{ Percent willing to utilize resource in future } \\
\hline Resource & $\begin{array}{c}\text { All } \\
\text { employees }\end{array}$ & $\begin{array}{c}\text { Civilian } \\
\text { employees }\end{array}$ & $\begin{array}{c}\text { Sworn } \\
\text { employees }\end{array}$ & $\begin{array}{l}\text { Fisher's } \\
\text { exact } p\end{array}$ & $\begin{array}{c}\text { All } \\
\text { employees }\end{array}$ & $\begin{array}{c}\text { Civilian } \\
\text { employees }\end{array}$ & $\begin{array}{c}\text { Sworn } \\
\text { employees }\end{array}$ & $\begin{array}{c}\text { Fisher's } \\
\text { exact } p\end{array}$ \\
\hline Chaplain & $1.6 \%$ & $4.5 \%$ & $0.5 \%$ & 0.06 & $13.5 \%$ & $12.1 \%$ & $14.1 \%$ & 0.83 \\
\hline $\begin{array}{l}\text { Critical Incident Stress } \\
\text { Debriefing }\end{array}$ & $64.9 \%$ & $25.8 \%$ & $78.9 \%$ & $<0.001$ & $52.6 \%$ & $30.3 \%$ & $60.5 \%$ & $<0.001$ \\
\hline $\begin{array}{l}\text { Counseling (inside } \\
\text { law enforcement) }\end{array}$ & $38.2 \%$ & $39.4 \%$ & $37.8 \%$ & 0.88 & $40.2 \%$ & $43.9 \%$ & $38.9 \%$ & 0.56 \\
\hline $\begin{array}{l}\text { Counseling (outside } \\
\text { law enforcement) }\end{array}$ & $47.8 \%$ & $54.5 \%$ & $45.4 \%$ & 0.25 & $49.4 \%$ & $51.5 \%$ & $48.6 \%$ & 0.77 \\
\hline Crisis Hotline & $2 \%$ & $3 \%$ & $1.6 \%$ & 0.61 & $10 \%$ & $13.6 \%$ & $8.6 \%$ & 0.24 \\
\hline Family Therapy & $31.9 \%$ & $34.8 \%$ & $30.8 \%$ & 0.12 & $34.3 \%$ & $36.4 \%$ & $33.5 \%$ & 0.76 \\
\hline $\begin{array}{l}\text { Friend (inside law } \\
\text { enforcement) }\end{array}$ & $78.9 \%$ & $57.6 \%$ & $86.5 \%$ & $<0.001$ & $68.1 \%$ & $53 \%$ & $73.5 \%$ & 0.003 \\
\hline $\begin{array}{l}\text { Friend (outside law } \\
\text { enforcement) }\end{array}$ & $81.7 \%$ & $80.3 \%$ & $82.2 \%$ & 0.71 & $70.9 \%$ & $69.7 \%$ & $71.4 \%$ & 0.87 \\
\hline Medications & $27.5 \%$ & $34.8 \%$ & $24.9 \%$ & 0.15 & $24.3 \%$ & $22.7 \%$ & $24.9 \%$ & 0.87 \\
\hline $\begin{array}{l}\text { Mindfulness/ } \\
\text { Meditation }\end{array}$ & $68.1 \%$ & $66.7 \%$ & $68.6 \%$ & 0.76 & $55.4 \%$ & $53 \%$ & $56.2 \%$ & 0.67 \\
\hline Minister/Faith Leader & $13.5 \%$ & $15.2 \%$ & $13 \%$ & 0.68 & $17.9 \%$ & $15.2 \%$ & $18.9 \%$ & 0.58 \\
\hline $\begin{array}{l}\text { Partner/Family } \\
\text { Member }\end{array}$ & $87.3 \%$ & $81.8 \%$ & $89.2 \%$ & 0.14 & $77.3 \%$ & $72.7 \%$ & $78.9 \%$ & 0.31 \\
\hline Peer Support & $29.5 \%$ & $13.6 \%$ & $35.1 \%$ & $<0.001$ & $31.1 \%$ & $16.7 \%$ & $36.2 \%$ & 0.003 \\
\hline Supervisor & $53.8 \%$ & $56.1 \%$ & $53 \%$ & 0.77 & $30.7 \%$ & $39.4 \%$ & $27.6 \%$ & 0.09 \\
\hline
\end{tabular}

Table 3. Percentage of all staff, civilian staff, and sworn staff who indicated that they had utilized specific mental health resources in their lifetime (left columns) or that they may or definitely would consider using specific mental health resources in the future (right columns). Significant group differences between civilian and sworn staff as calculated using Fisher's exact test are bolded. 


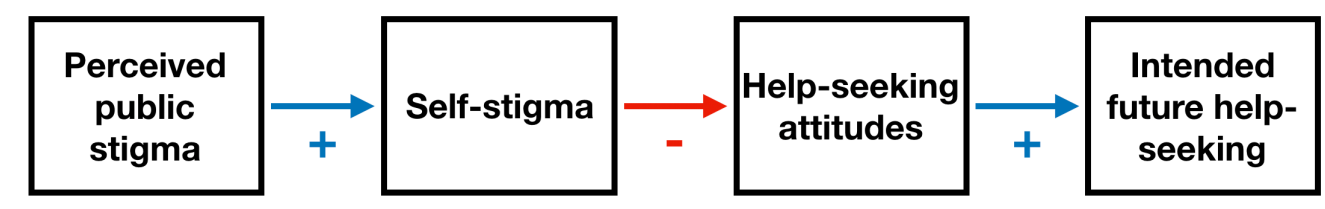

Figure 1. Theoretical model linking perceived public stigma, self-stigma, help-seeking attitudes and intended future help-seeking (based on Vogel et al., 2007).

A

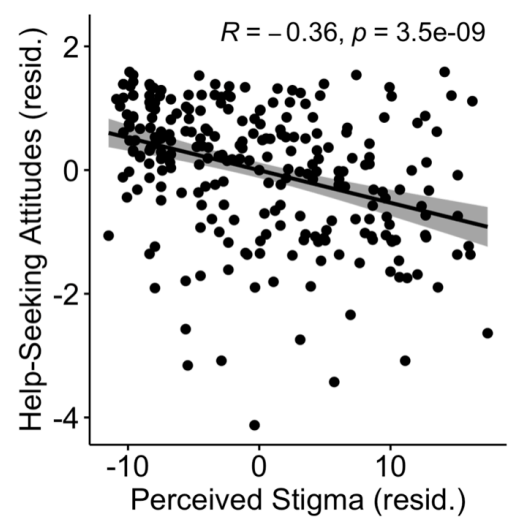

B

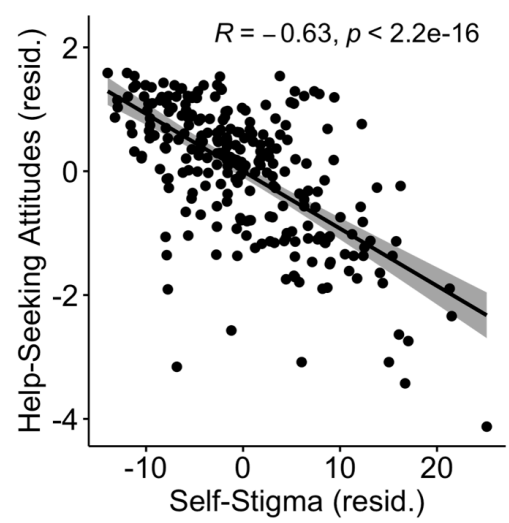

C

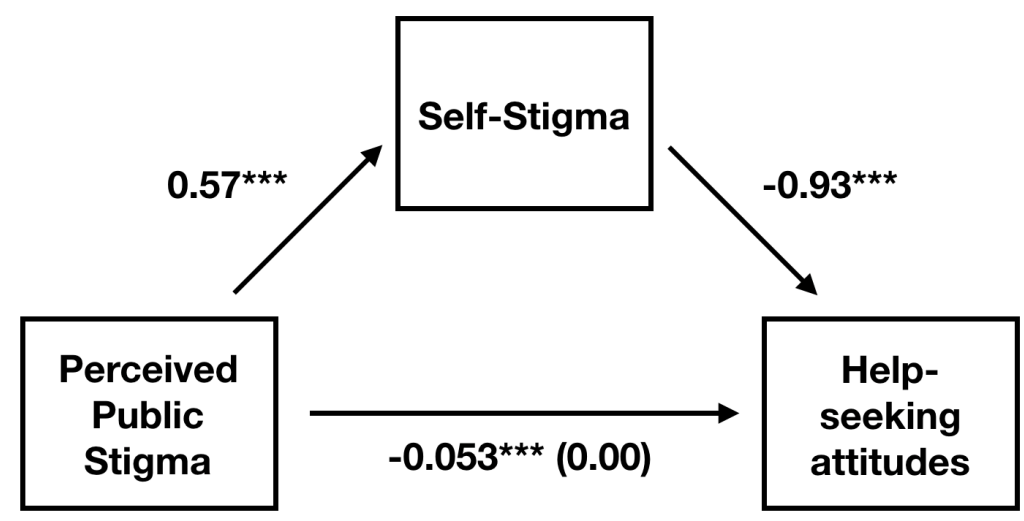

Figure 2. Increased perceived public stigma (A) and self-stigma of help-seeking (B) were each associated with more negative help-seeking attitudes, controlling for police agency, civilian/ commissioned status, gender, and years of work experience. (C) The relationship between perceived public stigma and more negative help-seeking attitudes was fully mediated by selfstigma of help-seeking. 
A

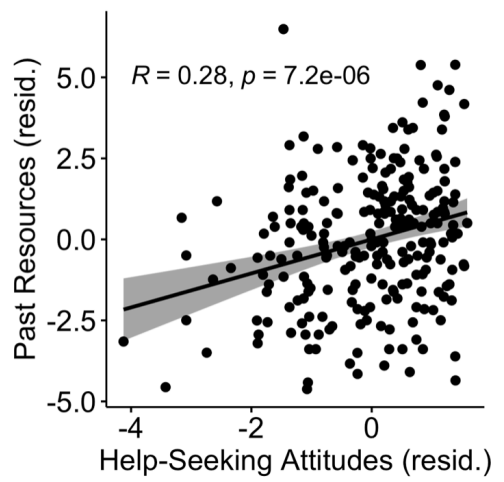

B

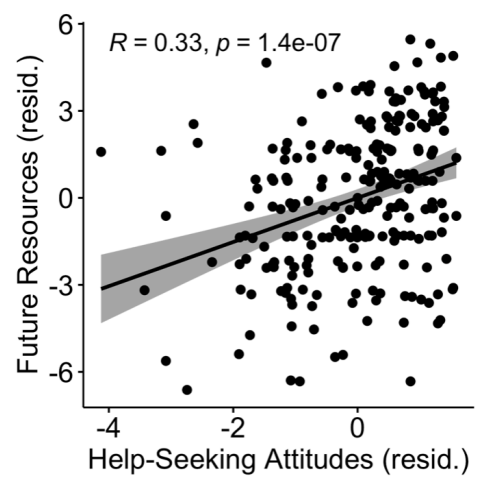

Figure 3. Controlling for police agency, civilian/commissioned status, gender, and years of work experience, more positive attitudes toward seeking help were associated with greater past utilization of mental health resources (A) and increased intentions to utilize mental health resources in the future $(B)$ 

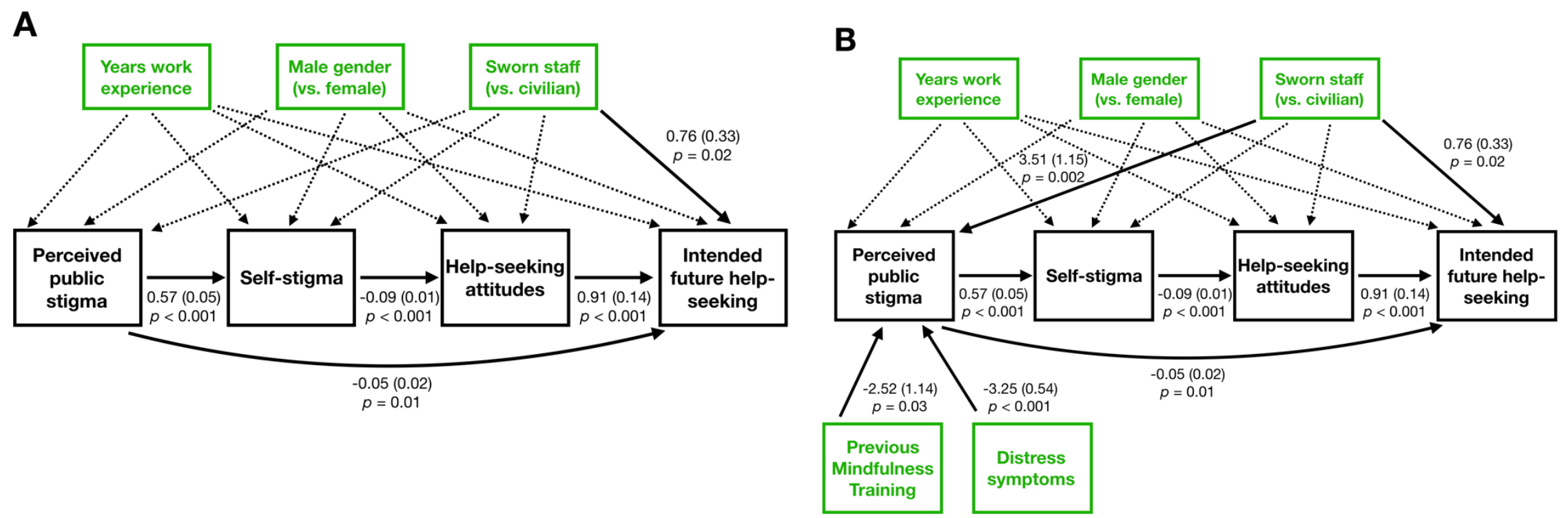

Figure 4. (A) Structural equation modeling provided evidence of a good fit for a path model linking increased perceived public stigma, increased self-stigma, more negative help-seeking attitudes, and decreased future intended help-seeking. There was a significant direct path from job classification to help-seeking intentions, with increased intended utilization of resources among sworn relative to civilian staff. (B) The inclusion of moderating variables indicated an indirect effect of greater psychological distress symptoms on decreased intended help-seeking via increased perceived public stigma. In contrast, previous mindfulness training contributed indirectly to greater future help-seeking via decreased perceived public stigma. The inclusion of moderators also resulted in a significant path between job classification and perceived public stigma, with sworn vs. civilian staff reporting greater stigma that resulted indirectly in decreased intended help-seeking. 